\title{
ODON: An On-Demand Security Overlay for Mission-Critical Applications
}

\author{
Jinu Kurian*, Ajay Kulkarni*, Hai Trong Vu*, and Kamil Sarac ${ }^{\dagger}$ \\ ${ }^{*}$ Dept. of Computer Science, University of Texas at Dallas, Richardson, Texas- 75080 \\ Email: $\{$ jinuk, hai.vu, ajay.kulkarni $\} @$ student.utdallas.edu \\ $\dagger$ Email: ksarac@utdallas.edu
}

\begin{abstract}
In this paper we consider the construction of a large-scale, highly available and secure overlay network to enable mission-critical communication between emergency personnel at a disaster area and their coordinating agencies across the Internet. This network is designed to be secure against network-based failures and external attacks including denial of service (DoS) attacks. We design several protocols for the effective operation of the network, including protocols for user access verification and the establishment of session credentials between the user and the target server. We verify these protocols theoretically for their security and evaluate the overall performance of the system with a combination of simulation and implementation
\end{abstract}

\section{INTRODUCTION}

In the event of an emergency situation or disaster event such as floods, earthquakes, hurricanes or terrorist attacks, it becomes imperative to expedite recovery operations to the affected sites in a timely manner. Therefore, it becomes vital that an effective communication medium is prevalent between various distributed disaster management organizations and emergency response personnel at the affected sites [1]. It is equally vital to consider the resiliency of this communication medium to interference in the form of attacks on the medium itself or general failures in the medium itself like outages and congestion.

In this paper we consider the problem of creating an ondemand overlay network for mission-critical communication requirements. Paramount in our design considerations is the establishment of a network which is resilient to failures in the network and cyberterrorism in the form of DoS and breakin attacks on the entities of the system. Our primary focus is to efficiently navigate the strict security and reliability requirements

Due to spatial limitations the paper has been significantly reduced in content. The reader is encouraged to refer to [2] for the full picture. The rest of the paper is organized as follows. In Section II we discuss the requirements and restrictions that we aim to meet in our design. In Section III we introduce the ODON overlay architecture, its management, operation and design rationale. In Section IV we look at the various functional components of ODON. In Section V we analyze the security properties of the system. Section VI evaluates the performance of the system under various conditions through a combination of simulations and implementation. Finally, Section VII concludes the paper.

\section{DESIGN REQUIREMENTS AND RESTRICTIONS}

In this section we describe the general requirements we consider in our design and some of the restrictions necessitated by the application context.

\section{A. Attacker threat model and operational assumptions}

We assume that the last-hop at the affected area has (possibly through an ad-hoc network) established communication to an access point in the wired Internet. We assume that there is a filter ring consisting of high-speed programmable routers at the destination domain which can be configured to filter out undesired traffic with ACLs or null routing. We also assume that the destination server has provided a set of users (during normal operating circumstances) with access control credentials (see Section IV-A for details) which can be used by the overlay nodes to verify the users authorization.

The attacker is assumed to have the ability to eavesdrop, record, replay or in other ways subvert the traffic in the system. It can also pretend to be any of the legitimate entities in the system and may be able to execute all or some of the steps in the access verification and token exchange protocols. The attacker may have knowledge of the location of every node in the ODON system and the target being protected. The attacker may be able to obtain legitimate credentials for the system. The attacker may have the ability to break-in and compromise any entity in the system except the protected destination. However it is assumed that the number of un-compromised nodes is at least twice the number of compromised nodes.

The attacker may have the ability to bring down through flooding attacks no more than one-third the number of nodes including the nodes currently in use in the system at any given time. The attacker can flood the destination server with unwanted traffic in an attempt to bring down the server. The attacker can also launch directed attacks against nodes being currently used in the system instead of randomly chosen nodes. We assume that the attacker can in worst case time $\mathrm{O}(\mathrm{n})$ * average rtt, correctly identify and specifically target an in-use overlay node. Here $\mathrm{n}$ is the number of overlay nodes in the system and rtt is the round trip time of a probe packet. The attacker does not have the ability to break into the destination server or its Key Distribution Center (KDC). It also does not have the ability to bring down the core of the Internet or the access point (there can be several) at the last-hop to the wired Internet.

\section{B. Design requirements}

High availability is one of the most crucial requirements of mission-critical/disaster management systems [3]. In the Internet, this translates to a high end-to-end packet-level availability which is defined as the ratio of the total number of packets sent to the number of packets received at the destination. The system should be highly available even during 


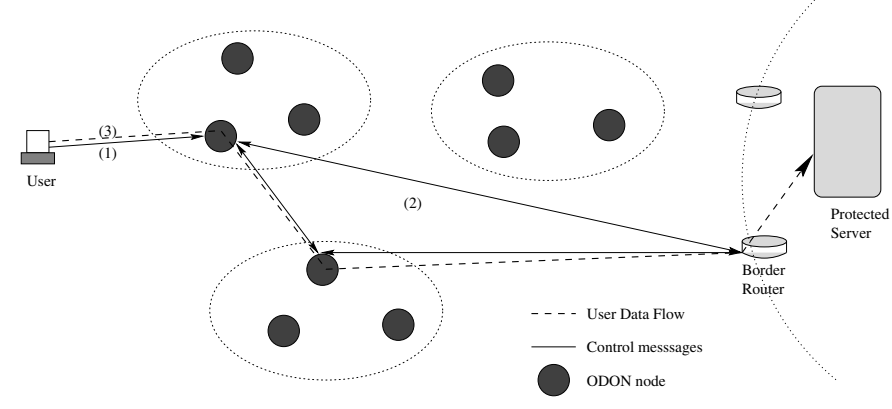

Fig. 1. Overlay topology: In the figure (1) is the access verification step, (2) is the session token exchange step, (3) is the flow of user data

aberrant circumstances like link failures, high congestion and DoS attacks on the system.

In general, the system needs to be secure against an attacker with capabilities as described in Section II-A. Security of the system therefore has to be three fold: 1) The system should be used only by authorized users of the system, 2) authorized but malicious users and/or compromised overlay nodes cannot break the system and 3) the system and the destination site must be resilient to DoS attacks.

In addition to these desired requirements, the application scenario also introduces various restrictions which introduce additional requirements. Fixed systems, i.e. a system with permanently live nodes, long lived secure connections, bandwidth guarantees or MPLS tunnels cannot be used. The system should be essentially dormant under normal operating circumstances and be live only when activated (on-demand) during a critical event. This is necessary because the system is to be utilized only during the emergency scenario and should not waste resources for its existence under normal circumstances. High end-to-end latency for communication should also be avoided. Though latency and user friendliness are not primary requirements, high latency limits the effectiveness and type of services available to efficiently coordinate between the user and the management agencies (e.g. a video conference between various departmental figureheads and the user).

\section{ODON: AN ON-DEMAND OVERLAY}

In this section we introduce the ODON overlay. We present the ODON architecture and an overview of its operation.

\section{A. Architecture}

Communication over ODON involves four entities: 1) users who are pre-authorized by the destination server and require effective communication with the destination for disaster recovery management, 2) overlay nodes deployed across the various domains in the Internet, 3) the destination server and 4) an ODON client installed at the users machine (see Figure 1). Unlike typical overlay networks, the overlay nodes do not form a pre-determined overlay network and do not forward user data across the overlay network. The overlay level connectivity in ODON is established on-demand and used only to exchange signalling and other management information between the overlay nodes. Based on the operational requirement, every node in ODON can act as either: 1) access node or 2) forwarding node. When acting as an access node, the overlay node serves as the authentication and access control entity for authorized users in the system. When acting as a forwarding node, the overlay node acts as a proxy. It verifies the users session token and forwards the user provided data through the filter ring to the destination in a one-hop source routing manner. On-hop source routing has been shown to give comparable performance results to multi-hop overlays in providing higher resiliency and response times to unicast based communication [4], [5]. In ODON we choose to use the onehop source routing approach because of its high performance and intrinsically simple operation. This is important because the on-demand nature of the system precludes complex routing schemes and established tunnels between overlay nodes. The ODON client is implemented as a proxy application in the users machine and at the destination server. All traffic going in and out of the system is intercepted by the ODON client and manipulated to work with the ODON overlay. The node organization in ODON is flat but separated into domains based on node proximity, i.e. node addressing is organized in the form domain.endsystem with nodes in a domain being aware of each other. This addressing scheme allows us to quickly search or disseminate information to all the nodes in a domain, for e.g. the partial keys during the access verification procedure (Section IV-A).

\section{B. Operation}

From a high-level perspective, the operation of ODON is as follows. In the event of an emergency scenario, an authorized user or a destination node chooses one of the overlay nodes to act as its access node. The authorized users credentials are verified (Section IV-A) by the access node which then executes the token exchange protocol (Section IV-B). After the users credentials are verified, the access node chooses a set of overlay nodes to act as forwarding nodes for the user and executes a three-way token exchange protocol between the user, the forwarding node and a border router at the destination domain. The objective of this token exchange protocol is to establish a session token and a session key to be used for the rest of the session between the user and the destination. At the completion of the token exchange protocol, the user is provided with a session token that is forwarded along with the user data and verified by the forwarding nodes before data forwarding. The user and the destination are also provided with a session key that is used to provide integrity and confidentiality to user traffic.

The ODON client at the user end then replicates and forwards user traffic along with the session token to the chosen forwarding nodes. The forwarding nodes verify the users session token and forward the users traffic through the filter ring towards the destination. During the course of the session, a chosen forwarding node initiates another token exchange session between the user and another distinct set of overlay nodes. This allows the ODON client to rapidly switch from a poorly performing forwarding node to a new forwarding node 
when required.

The freedom to switch between any pair of overlay nodes is a unique and vital component of the ODON architecture. It allows the user to avoid poorly performing nodes which may be congested or subject to DoS attacks. It also helps in dealing with possibly compromised forwarding nodes as we will see in Section V. To decide when to switch to a new forwarding node, every ODON client measures two metrics: i) RTT between the user and the destination per forwarding node and ii) decay, the total time a particular forwarding node has been in use during a given session. Based on these measured metrics, the ODON client maintains two additional metrics: i) Estimated RTT measured as estrtt $=0.9^{*}$ estrtt $+0.1^{*} \mathrm{rtt}$ and ii) Average decay. The ODON client switches to a new forwarding node if the measured RTT $=\alpha *$ estrtt or the decay is greater than the average decay, where $0 \alpha 1$ is the scaling factor.

\section{Functional Components}

In this section we discuss in detail the components that make up the operation of the system. We discuss the protocols used by ODON for user access verification and session token exchange and traffic forwarding by the forwarding node after the session is established.

\section{A. Access Verification}

The access verification step consists of the access node retrieving an authentication token installed in the users machine and verifying it based on user entered information. This authentication token is a single-use token installed in the users machine or provided in a storage device to the user by the KDC at the destination side during normal operating circumstances. The access verification procedure at the access node effectively pushes a distributed filter deep into the network away from the destination. It ensures that the connection establishment process by itself cannot be used by an attacker for DoS attacks. It also helps protect the destination from directed flooding based DoS attacks. Finally it establishes a shared key between the user and the destination ensuring that only authorized users can utilize the ODON overlay and that the end-to-end communication is secure and confidential.

Key Distribution: During normal operating circumstances a KDC at the destination site generates a set of encrypted partial keys kpi for distribution to the overlay nodes. The keys, like node addresses are indexed by domain with a range of key values being assigned to nodes based on their domain. This allows the access node to easily search for the partial keys by contacting any node within the desired domain. The partial keys are used to generate a set of encryption keys Ki. In general a key $\mathrm{Ki}=\mathrm{kpa}$ xor $\mathrm{kpb}$ xor ...kpk such that no two partial keys belong to the same node. The rationale behind distributing the keys in this manner is to avoid the collateral damage that can occur if any of the overlay nodes are compromised albeit at a loss in performance. This performance hit can however be avoided at the cost of higher complexity and less scalability by using a $(\mathrm{k}, \mathrm{n})$ threshold key scheme [6] to distribute the keys. These encryption keys are used in the generation of a set of authentication tokens that are distributed to the authorized users. These authentication tokens will (see Section IV-A) be used for the access verification at the access nodes.

Structure and security of authentication token: The structure of the installed token is shown in figure 2. The token has 8 fields, Domain, Exp(iry), Key index, Data, U(ser)name, $\mathrm{P}($ ass)w(or)d, Seal and Sig(nature). The domain field contains the domain (i.e. the destination server) for which the token was issued and is valid for. Exp contains an explicit expiration time for the token in cleartext. Key index contains an index which references the partial keys used to generate the encryption key used in the creation of the token. Data field contains other optional information which can be used to specify additional information about the users access privileges. Pwd and Uname are encrypted versions (using the encryption key) of the users username and password used for access verification at the destination. These credentials are temporary and generated by the KDC only for use in the ODON system. Seal is a keyedhash of the entire token keyed with a key derived from the encryption key and finally the Sig field is a signature created by the KDC over all the token fields except the Seal.

The authentication token described has some important properties that make it secure and non-transferable. The Domain field binds the token to a single domain. Even if the token is intercepted by an adversary, it is not useful without the username and password of the user. If required, the token can also be bound to a single user machine (for example using a combination of fields like BIOS checksum, MAC address, operating system version etc.) which ensures that it cannot be distributed to create an attack. The Seal field ensures that the token has not been altered in any manner by the user or an attacker. Since the seal includes the Expiry and Id fields as part of the hash, the user cannot modify the token to extend its validity or reuse an expired/revoked token. The Sig field created by the KDC ensures that the token cannot be manufactured by an adversary. Finally the partial keys used for creating the token are expired by the access node after the authentication process is completed by disseminating this information to the nodes which returned the partial keys during the search. This ensures that the token is invalid after its first use.

Authentication and access control: In ODON the authentication and access control procedure is done simultaneously when the user presents his token and provides his credentials. The protocol is displayed in figure 2 . In the figure, A refers to the user, S refers to the access node, SKey(AS) is a shared key between $\mathrm{A}$ and $\mathrm{S}$ derived from the password of user. After obtaining the token and decrypting the pwd field, the access node is also able to derive this key from the password. As can be seen in this process, the user by providing its credentials and having them verified authenticates itself and establishes its identity. By virtue of it possessing a valid token, it additionally establishes its access privileges and thus completes the access verification step. 


\section{B. Token exchange protocol}

The token exchange protocol is a three way protocol initiated by the access node after the user token is verified to establish a session token. This session token is shared by the user, its forwarding node and the border router at the destination. Additionally it also establishes a shared secret key and nonce between the user and the destination server. The protocol is displayed in figure 3. In the figure, A is the user, B is the destination server, $\mathrm{S}$ is the access node, $\mathrm{O}$ is a forwarding node and $\mathrm{R}$ is the border router/KDC at the destination. In this process we assume that the forwarding node chosen are the nodes which returned the partial keys during the key search in the access verification procedure.

This simplifies the key exchange process so that the shared key SKey(SO) can be derived from the partial key returned. The shared key SKey(SR) is assumed to be a long term shared key between the nodes in a domain and the border node and Skey(AR) is a shared key between the user and the KDC created based on the users credentials at the KDC. The protocol proceeds as follows. The access node contacts the border router which returns the session token and a ticket consisting of a session key and nonce encrypted with the shared key between the user and the KDC. The access node then contacts the forwarding node(s) with the session token. The session token is modified (with the Seal2 field) by the forwarding node and returned to the access node. Finally the access node returns the token and the ticket to the user for its session. The structure of the session token is shown in figure 3. Domain and Exp are similar to the access token. The ID field binds the token to a single forwarding node. The Seal2 field is created by the forwarding node over the data in the token while the Seall field is created by the border router. Since the token is valid only at a single forwarding node and unusable after the session is completed due to its short expiration time, replay and man-in-the-middle attacks are avoided here. The token exchange protocol is repeated with one of the active forwarding nodes acting as the initiator with another set of nodes for fast node switching. The ODON client at the user receives the session token, a nonce and a session key at the end of the token exchange protocol. The ODON client encrypts the data provided by the user with the session key along with the nonce and sends it to the forwarding node along with the session token. The process is repeated if there are multiple forwarding nodes so that the data is replicated between all forwarding nodes. A forwarding node upon receiving the user data initially verifies the session token. It does so by recomputing the Seal2 field and the ID field. If valid, it forwards the data towards the destination server through the border router. The router again verifies the token by recomputing the Seal2 field and if verified forwards the traffic to the destination server. The reverse side traffic also follows the same path through the forwarding node to the user. Note that since the session token is valid only at a single forwarding node, the token can be sent in cleartext and is useless to an attacker.

\begin{tabular}{|c|c|c|c|c|c|c|c|}
\hline Domain & Key Info & Uname & Pwd & Exp & Data & Seal & Sig \\
\hline \multicolumn{8}{|c|}{$\begin{array}{l}1 \mathrm{~A} \rightarrow \mathrm{S}:\{\text { Token, A, B, S, Uname, Pwd, n1 }\}\{\operatorname{SKey}(\mathrm{AS})\} \\
2 \mathrm{~S} \rightarrow \mathrm{A}:\{\mathrm{B}, \mathrm{A}, \mathrm{S}, \mathrm{n} 1+1\}\{\operatorname{SKey}(\mathrm{AS})\}\end{array}$} \\
\hline
\end{tabular}

Fig. 2. Structure of the Access Token and Access Verification Protocol

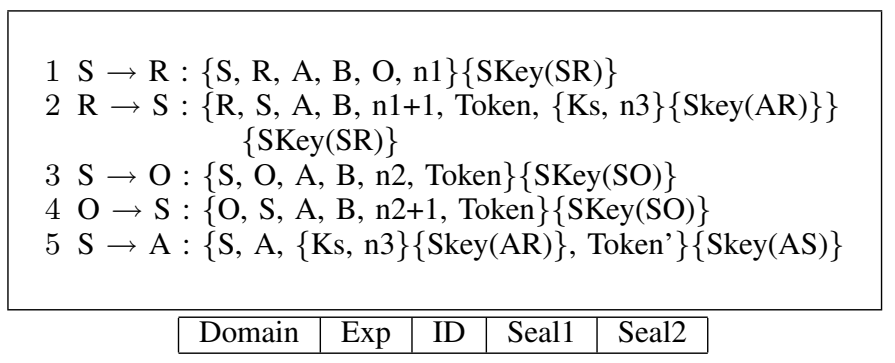

Fig. 3. Token exchange protocol and Token.

\section{SEcurity Analysis}

In this section we we use theoretical protocol modeling techniques to analyze the security of the access verification and token exchange protocol described in the previous section. Finally we discuss the security properties of the system and its effectiveness in dealing with compromised nodes.

\section{A. Theoretical perspective}

In this subsection we use formal verification mechanisms to evaluate the correctness of our two proposed protocols.

The language of Communicating Sequential Processes (CSP) [7] can be used to describe any system with agents that communicate by passing messages between each other. Among other applications like software engineering, parallel processing and distributed systems, CSP also allows for the formal description of entities in an authentication protocol and the messages used in the protocol. CSP has been used extensively in the analysis of security protocols including the breaking and fixing of the Needham-Schroeder protocol [8], RADIUS [9] and many others [10].

Details about the verification for the protocols can be found in [2]. Specifically, we model the interactions between the participating entities as communications between a set of sequential processes. This allows us to verify that the shared secrets (password, nonce, session key) are shared only between the participants of the protocol and not to an intruder.

\section{B. Security properties of the system}

In this subsection we discuss 5 important security properties possessed by the ODON system. As we will show, these properties combine to make the system secure against many possible attacks.

Property 1: Dropping or delaying traffic by overlay nodes is detected by the ODON client. This property follows directly from the node cycling property of ODON. The ODON client at the user site monitors its chosen forwarding nodes for performance. If the forwarding node is dropping or delaying 
packets (either maliciously or due to network conditions), the ODON client forces a switch to another node.

Property 2: There are at least $2 \mathrm{k}+1$ forwarding nodes, where $\mathrm{k}$ is the maximum possible number of compromised node. The ODON client always replicates its traffic among multiple forwarding nodes. Such an assumption on the number of compromised nodes is used in Byzantine resiliency calculations. Since each forwarding node is in use for a short period of time, if a node recovery mechanism is present on the suspicion of a compromised node, the assumption is also practically valid. Property 3: Access tokens and session tokens are single use and additionally session tokens are valid only at a single forwarding node. Since the key generating the token is expired after a successful authentication and session tokens are valid only for a very small period of time, they are both single use. Additionally the session token is tied to a single forwarding node by the Seall field.

Property 4: The signature field in the access token can be verified by any overlay node. The signature is created by the destination KDC. Its assumed that all overlay nodes are provided with a valid certificate for the KDC.

Property 5: The border router(s) at the destination mediate all session requests to the target.

\section{EVALUATIONS}

\section{A. Motivation}

In this section we present simulation based analysis of the performance of the ODON system. In particular we aim to demonstrate that the requirements specified in Section III are met in our design of the ODON architecture. We measures the performance of the system across three specified requirements: 1) High Availability, 2) DoS resistance, 3) End-to-end latency introduced by the system.

\section{B. Simulation setup}

We use the BRITE topology generator to create a hierarchial top-down topology consisting of 10 ASs with 50 nodes in each AS for a total of 500 nodes. There are 10 users and 20 overlay nodes randomly distributed across all the domains in the topology. The destination server is randomly chosen from the remaining nodes. The experiments are conducted under varying environmental conditions including: i) emergency operating environment which is characterized by flash crowds crossing the network and random failures ii) Flooding based DDoS attacks directed at the target server and overlay nodes and iii) DDoS attacks on the connection establishment process. All our simulations are repeated three times to account for the randomness and the results are averaged over all three runs.

\section{Metrics}

Packet level availability: Availability is defined as the ratio of the total number of packets sent to the number of packets received at the destination. This includes lost and retransmitted packets. For ODON since the traffic is replicated by two, if either or both of the replicated packets are received, the number of received packets is assumed to be two for two sent packets.
Response time: Response time is a measure of the time in msec for a request sent by the user to be responded to by the server.

\section{Emergency environment}

To model this scenario, in addition to the background traffic as in the normal case at a lower rate of $2 \mathrm{Mbps}$, we create flash events targeting various distributed servers in the topology [11]. We create this flash event by adding a new sender to a given destination every 0.01 seconds in the background traffic till it peaks, maintaining it for 2 seconds and ramping down till the sending rate is the base $2 \mathrm{Mbps}$. The flash events are repeated with more added senders with a 1 second gap in between each event to model larger and larger flash events.

\section{E. DoS attacks}

Denial of service attacks can target either the destination or the the nodes in the system for the non-unicast cases. To create DoS attacks, the attacking senders send traffic at 10 Mbps from each sender to the destination and to the currently in-use overlay node for the non-unicast cases. In the unicast case the attack is concentrated on the target alone while in the other cases, the attack traffic directed at the target is dropped at least 2 hops away from the final target. Based on our previous discussion in Section II-A, directed attacks on the forwarding node occur within a period exponentially distributed between 100 and $250 \mathrm{~ms}$ from the time the node becomes in use.

\section{F. DDoS attacks on the connection establishment process}

These experiments directly compare the connection establishment process of OverDoSe with ODON. In the case of OverDoSe, the attacker generates a large number of connection establishment requests from a large number of hosts such that the rate generated by each host is low enough to be not filtered out by the overlay node. This attack is replicated across multiple overlay nodes. Every request made by the attackers may either be filtered out by the overlay node if the total volume of traffic through the node is too high, or transmitted to the server which is then required to verify it and admit or filter out the request. The filtering out of requests by the overlay node has the effect of dropping legitimate requests also which use the same overlay node. This also increments the difficulty of the puzzles generated by the server throttling the sending rate of both attacker and legitimate user and additionally causes legitimate users to be preferentially discarded because the attackers solve equivalently high level of puzzles. In the case of ODON, all attackers are assumed to initially possess a legitimate token (which should never happen in a realistic scenario). However since the token is expired after initial use, the attack cannot be sustained once the session is required to be renewed. Figures 4(a-d) show our simulation results. Fig 4(a) and 4(b) show that the availability of the system is much higher in all the overlay based cases in comparison to the unicast case. The ODON case in particular maintains a very high availability metric even under DoS attacks and the flash events during the emergency scenario. Fig 4(c) show the 


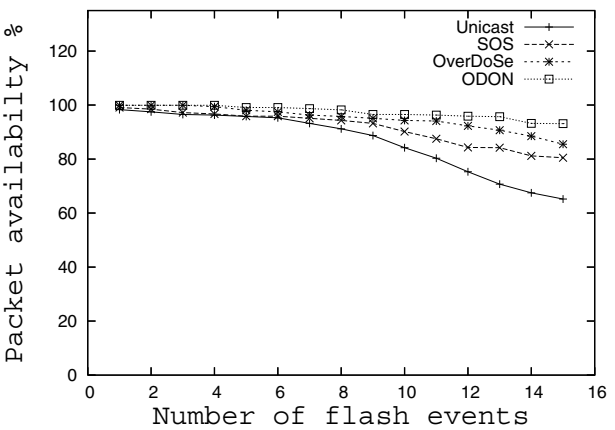

Fig. 4(a). Availability under emergency conditions.

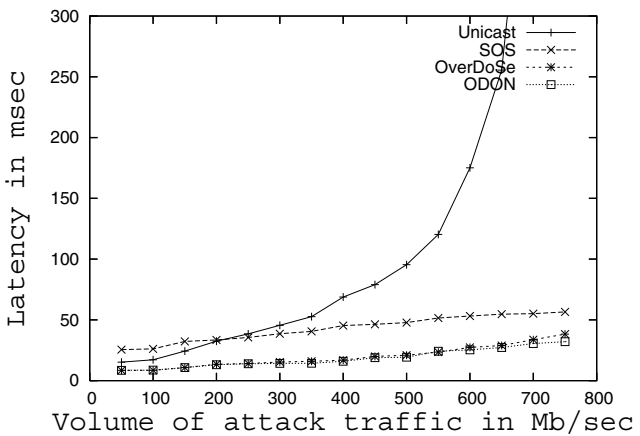

Fig. 4(c). Response time during DoS attacks.

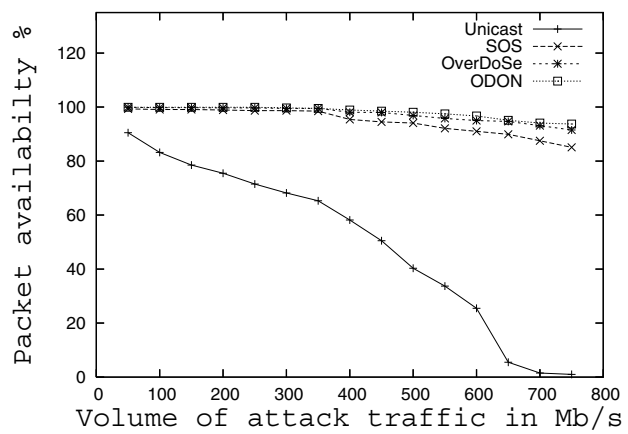

Fig. 4(b). Availability during DoS attacks.

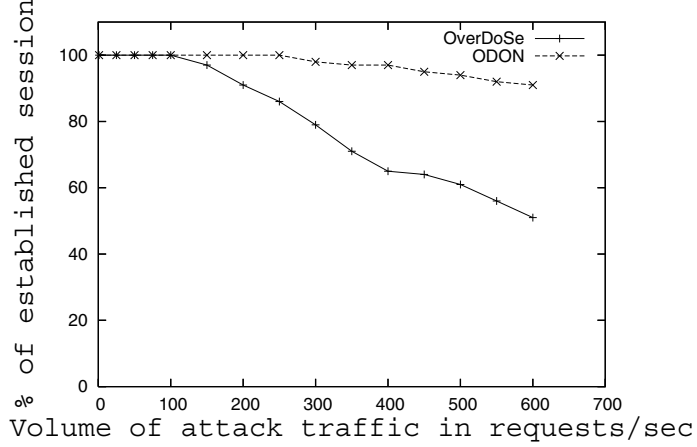

Fig. 4(d). Percentage of established sessions under DoS attacks. total response times of the system under varying conditions and varying levels of traffic. For lower loads in the system the unicast traffic shows better latency than the overlay based cases. However as the load increases, OverDoSe and ODON show a very consistent value of latency as opposed to the unicast case. SOS suffers from a large overhead introduced by its circuitous routing. ODON again outperforms OverDoSe because of the larger number of concurrent paths used and switching of forwarding nodes before they become overloaded. Fig 4(d) shows the percentage of completed legitimate session requests under a distributed request flood attack. As can be seen from the figure, OverDoSe suffers from a large number of dropped sessions due to the overloaded server. ODON filters out most of the spurious requests at the overlay level itself maintaining a near perfect session establishment rate. Our simulations demonstrate the effectiveness of ODON under loaded network conditions and DoS attacks on the system. It performs consistently better than the unprotected cases and comparable work in DoS-resistant overlay networks.

\section{CONCLUSION}

In this paper we considered the problem of creating a secure, highly available network for mission-critical applications. We believe that our work is the first of its kind which deals exclusively with the nuances of the mission-critical application scenario. For ease of deployment and to obtain high availability we designed the system as an overlay network with forwarding nodes. We designed and verified the correctness of the required access verification and session establishment protocols and also evaluate the performance of the system with a combination of simulation and implementation. Our results show that the ODON system is highly effective in meeting the stringent requirements of the application and performs better than comparable work in related areas.

\section{REFERENCES}

[1] K. Carlberg and R. Atkinson, "General requirements for emergency telecommunication service," February 2004.

[2] J. Kurian, H. T. Vu, A. Kulkarni, and K. Sarac, "Odon: An on-demand overlay network for mission critical applications," Univeristy of Texas at Dallas, Tech. Rep., May 2008, available at http://utd.edu/jinuk/Icccn09.pdf.

[3] M. Liotine, Mission-Critical Network Planning, 1st ed. Artech House Publishers, October 2003.

[4] D. Andersen, H. Balakrishnan, M. Kaashoek, and R. Morris, "Resilient overlay networks," in Proceedings of 18th ACM SOSP, Banff, Canada, October 2001.

[5] K. Gummadi, H. Madhyastha, S. D. Gribble, H. M. Levy, and D. J. Wetherall, "Improving the reliability of internet paths with one-hop source routing," in Proceedings of 6th USENIX Symposium on Operating systems design and implementaion (OSDI), San Fransisco, CA, USA, December 2004.

[6] A. Shamir, "How to share a secret," Communications of the ACM, vol. 22 , no. 11 , pages $=612-613$, month $=$ November, 1979 .

[7] T. Hoare, Communicating Sequential Processes. Prentice Hall, 2004.

[8] G. Love, "Breaking and fixing the needham-schroeder public-key protocol using csp and fdr," Lecture Notes in Computer Science, vol. 1055, pp. 147-166, 1996.

[9] I.-G. KIM and J.-Y. CHOI, "Model checking of radius protocol in wireless networks," IEICE Transactions on Communications, pp. 397408.

[10] S. Schneider, "Verifying security protocols: an application of csp," in Proceedings of 25 Years of CSP, London, England, July 2004.

[11] National Research Council (U.S.A), The Internet Under Crisis Conditions: Learning from September 11, 1st ed. National Academies Press, January 2003. 